\title{
Xantoma del tendón de aquiles bilateral: Debut de xantomatosis cerebrotendinosa
}

\section{Bilateral Achilles Tendon Xanthoma: Cerebrotendinous Xanthomatosis Debut}

\author{
María Dolores Fernández-Ruiz ${ }^{10}$ Lorena Moril-Peñalver ${ }^{1} \quad$ Alexandra Sevilla-Monllor ${ }^{1}$ \\ María Tíscar García-Ortiz ${ }^{1 \odot}$ Alejandro Lizaur-Utrilla ${ }^{2}$ Fernando López-Prats ${ }^{1,2}{ }^{1}$ \\ 1 Departamento de Cirugía Ortopédica y Traumatología, Hospital \\ Address for correspondence María Dolores Fernández-Ruiz, MD, \\ General Universitario de Elche. C/ Almazara, Elche (Alicante), España \\ 2 Departamento de Traumatología y Ortopedia, Universidad Miguel \\ Comunidad Autónoma, 37, La Copa de Bullas, Murcia, 30189, España \\ Hernández, San Juan de Alicante, España \\ Rev Chil Ortop Traumatol 2020;61:112-115.
}

\section{Resumen}

\section{Palabras clave}

- xantoma

- tendón de Aquiles

- xantomatosis cerebrotendinosa

\section{Abstract}

Objetivo Presentación de un inusual caso de xantomatosis cerebrotendinosa en un paciente de edad cuya primera manifestación fueron xantomas bilaterales del tendón de Aquiles.

Material y métodos Mujer de 62 años, que presenta tumoraciones sólidas y polilobuladas, en la cara posterior de ambos tendones de Aquiles de 8 años de evolución. El diagnóstico se realizó mediante el hallazgo de hiperlipidemia y estudio genético. Se realizó la exéresis quirúrgica parcial de las tumoraciones.

Resultados A los 5 años de la cirugía del pie izquierdo y 4 años del pie derecho la paciente estaba asintomática. Presentaba una fuerza para la flexión plantar bilateral de $5 / 5$, pudiendo caminar y subir escaleras sin molestias. Presentaba una escala AOFAS de 85 y 90 puntos en el pie izquierdo y derecho, respectivamente. No hubo recidivas.

Discusión Los xantomas son depósitos de colesterol en el tejido conectivo de la piel, tendones o fascia, como resultado de una hiperlipoproteinemia. La importancia del caso radica en su sospecha diagnóstica, ya que la xantomatosis cerebrotendinosa suele manifestarse en pacientes de menos de 30 años de edad y en los que se ha recomendado la resección radical de las tumoraciones, e incluso del tendón, debido a las frecuentes recidivas.

Conclusión En pacientes de mayor edad con lesiones que infiltran el tendón, se puede optar por un tratamiento menos agresivo con un buen resultado clínico.

Nivel de evidencia IV

Objective Presentation of an unusual case of cerebrotendinous xanthomatosis in an elderly patient whose first manifestation was bilateral Achilles tendon xanthomas.

Material and methods 62-year-old woman presenting solid and polylobed tumors on the posterior aspect of both Achilles tendons for eight years. The diagnosis was made by means of hyperlipidemia and a genetic study. Surgical partial excision of the tumors was performed. received

August 19, 2020

accepted

August 25, 2020
DOI https://doi.org/

10.1055/s-0040-1719020.

ISSN 0716-4548.
Copyright $\odot 2020$ by Thieme Revinter

Publicações Ltda, Rio de Janeiro, Brazil
License terms

()ㅇㅇ $\Theta \circledast$ 


\section{Keywords \\ - xanthoma \\ - achilles tendon \\ - cerebrotendinous xanthomatosis}

Results Five years after surgery on the left foot and four years after the right foot, the patient was asymptomatic. Bilateral plantar flexion force was $5 / 5$, The patient was able to walk and climb stairs without discomfort. AOFAS score was 85 and 90 on the left and right feet, respectively. There were no recurrences.

Discussion Xanthomatosis is a genetic alteration with deposits of cholesterol in connective tissue of the skin, tendons or fascia, because of hyperlipoproteinemia. The importance of the present case lies in its diagnostic suspicion, since cerebrotendinous xanthomatosis manifests usually in patients under 30 years of age and in whom radical resection of tumors, and even of the tendon, has been recommended due to frequent recurrences .

Conclusion In older patients with tumors that infiltrate the tendon, a less aggressive treatment can be chosen with a good clinical result.

Level of evidence IV

\section{Introducción}

Los xantomas son depósitos de colesterol en el tejido conectivo de la piel, tendones o fascia. Se manifiestan clínicamente como nódulos duros y profundos cubiertos por piel normal. Aparecen en cualquier localización, siendo más frecuentes en tendones extensores de dedos, codo, rodilla y, especialmente, en el tendón de Aquiles.

Están altamente asociados con la hiperlipidemia que se puede manifestar como sitosterolemia, hipercolesterolemia familiar o xantomatosis cerebrotendinosa. ${ }^{1}$ Esa última, es una enfermedad autosómica recesiva derivada de la mutación del gen del esterol 27-hidroxilasa (CYP27). Tiene como consecuencia la acumulación de colesterol y colestanol en el cerebro, los ojos, los tendones y otros tejidos. ${ }^{2} \mathrm{Se}$ diagnostica en base a hallazgos clínicos, bioquímicos, pruebas de imagen y análisis genético molecular (gen CYP27A1). Suele aparecer en los primeros 30 años de vida, con una presentación clínica variable, como síntomas neurológicos (epilepsia, depresión, ataxia y deterioro mental), cataratas, aterosclerosis y xantomas tendinosos.

Los xantomas de vainas tendinosas afectan principalmente al tendón de Aquiles y con menor frecuencia a otros tendones. ${ }^{3-6}$ Dado que presentan la misma fisiopatología que el depósito de colesterol en las arterias, los xantomas representan un marcador de alto riesgo para la enfermedad cardiovascular, ${ }^{4}$ y son una indicación de tratamiento de los niveles séricos de colesterol. ${ }^{7}$ Además, la reducción en el tamaño del xantoma que aparece en el tendón, coincide con una reducción de los niveles séricos de colesterol, una reducción del grosor íntima-media de la carótida y una reducción de la placa ateromatosa. ${ }^{8}$

Presentamos el caso de una paciente de 62 años, con un debut tardío de xantomatosis cerebrotendinosa, que tiene como primera manifestación la aparición de xantomas bilaterales en el tendón de Aquiles.

\section{Caso Clínico}

En enero de 2015 una mujer de 62 años de edad, es remitida a las consultas de Cirugía Ortopédica por la aparición espontánea de tumoraciones en ambos pies de ocho años de evolución. A la exploración, se apreciaban tumoraciones de consistencia sólida que adelgazan la piel en la cara posterior de ambos tobillos, dolorosas a la presión y dificultando la deambulación (-Fig. 1). La paciente estaba en tratamiento con estatinas por dislipemia desde hacía 3 años.

La resonancia magnética (RM) (-Fig. 2) mostraba tumoraciones poliglobuladas de uno a cinco centímetros de diámetro en contacto con el tendón de Aquiles, tendones peroneos y fascia plantar en el pie izquierdo; y en el tendón de Aquiles en el derecho. En los análisis bioquímicos, se detectaron aumentos de los niveles séricos de colesterol (233 mg/dL) y LDH (156 mg/dL). La RM cerebral no mostró hallazgos patológicos de interés El diagnóstico de xantomatosis cerebrotendinosa fue confirmado mediante un estudio genético, hallándose dos variantes patogénicas en heterocigosis como responsables de la enfermedad: c. $1183 C>$ T, p.(Arg395Cys), rs121908096; y c.1213C > T, p. (Arg405Trp), rs573951598. Debido a las molestias funcionales, se decidió la exéresis quirúrgica.

En el pie izquierdo, mediante un abordaje longitudinal paratendinoso medial se extirparon las múltiples tumoraciones cercanas al tendón de Aquiles. La tumoración proximal estaba adherida al peritendón siendo necesaria la resección del mismo. La tumoración más distal envolvía e infiltraba la totalidad del tendón de Aquiles y la piel, obligando a realizar una resección parcial de la misma (-Fig. 3). Mediante un segundo abordaje longitudinal en el arco plantar interno, se realizó la exéresis parcial de la fascia plantar. Postoperatoriamente, el tobillo se inmovilizó por cuatro semanas, seguido de fisioterapia con carga completa. Se continuó el tratamiento con estatinas sistémicas, e infiltraciones locales con ácido desoxicólico. El análisis anatomopatológico confirmó el diagnóstico.

El tobillo derecho fue operado un año después. Mediante un abordaje longitudinal paratendinoso central sobre el tendón de Aquiles, se observó que la tumoración infiltraba distalmente la totalidad del tendón de Aquiles, sin objetivarse tejido sano. Por ello, solo se realizó exéresis parcial de la tumoración. 

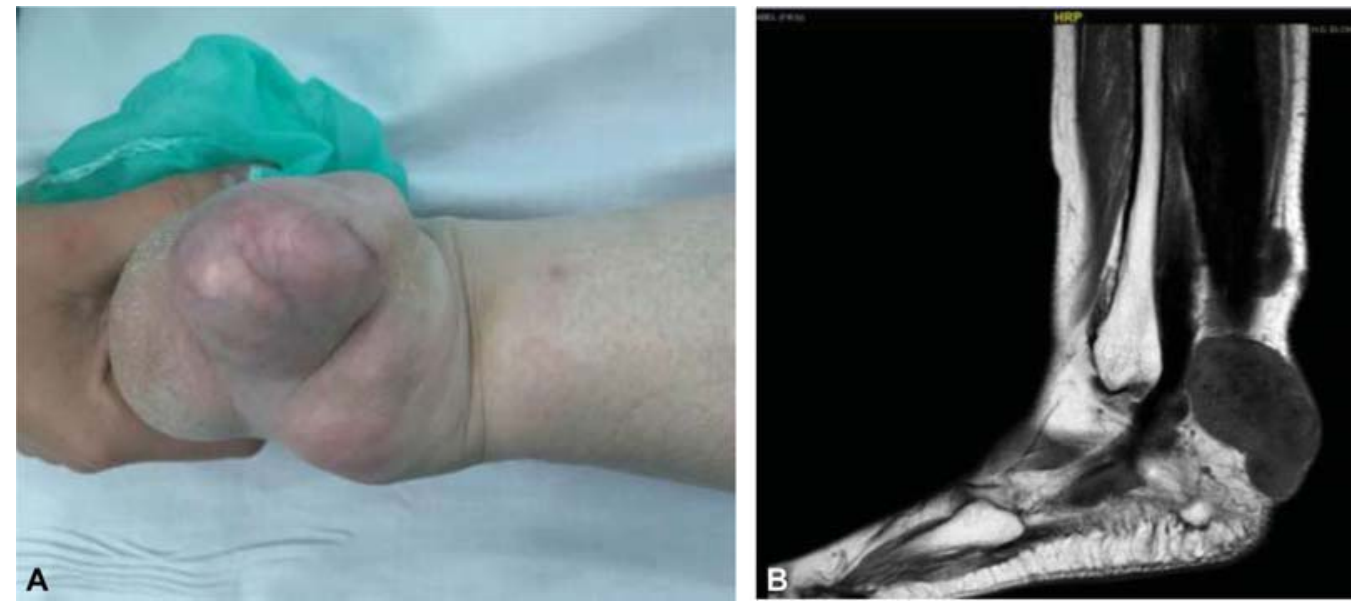

Fig. 1 Xantomas de tendón de Aquiles en el tobillo y pie derecho. A) Aspecto preoperatorio. B) Resonancia magnética.
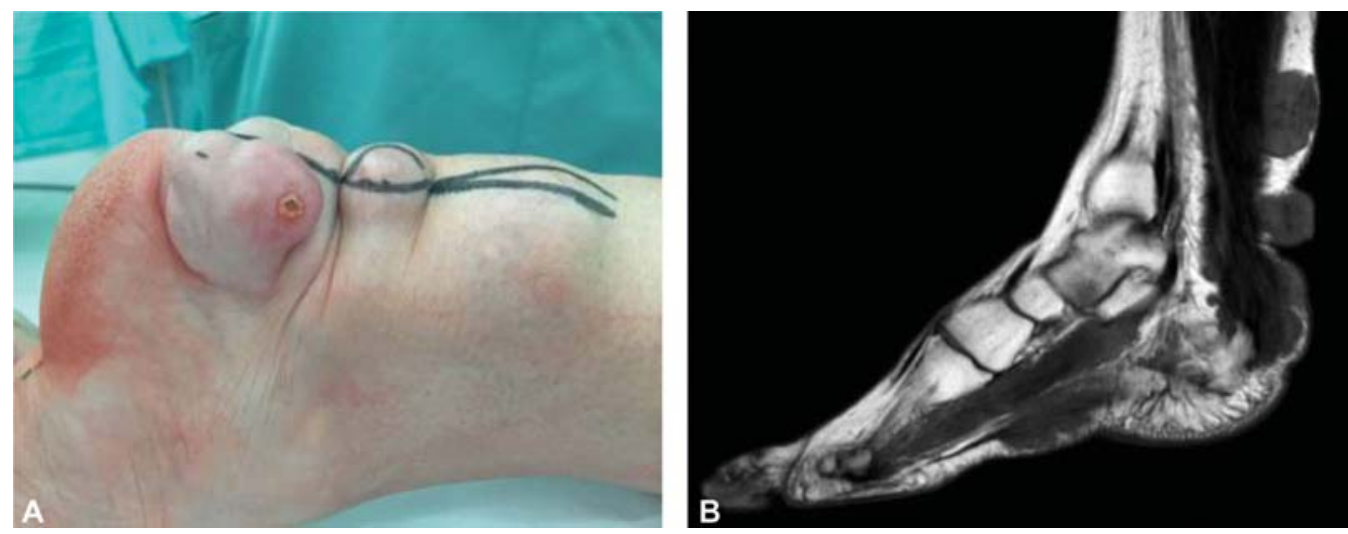

Fig. 2 Xantomas de Aquiles en el tobillo izquierdo. A) Aspecto preoperatorio. B) Resonancia magnética, apreciándose afectación del tendón y de la fascia plantar.

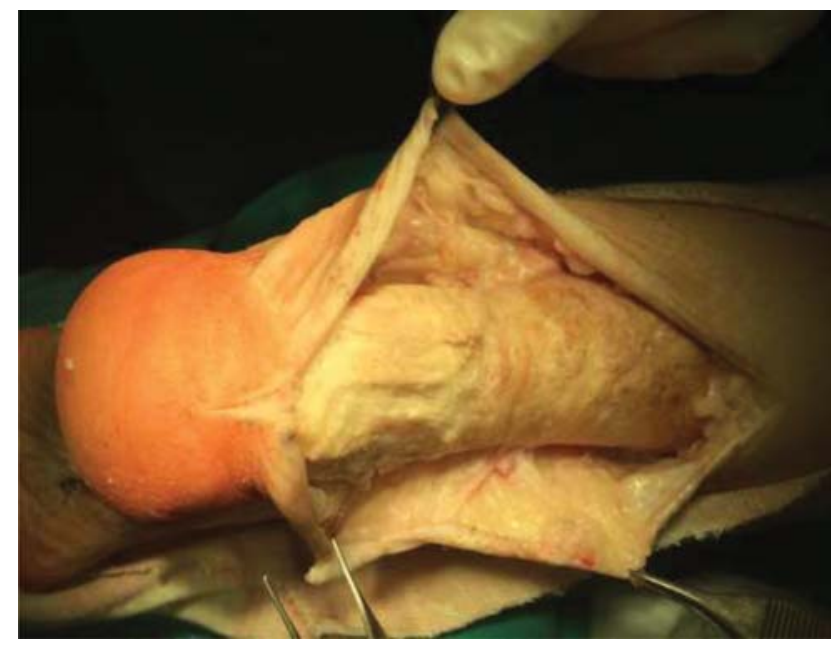

Fig. 3 Imagen intraoperatoria de xantomas en tendón de Aquiles izquierdo.

Preoperatoriamente, la escala AOFAS en el tobillo izquierdo era de 65 puntos, con recuperación funcional a un año preoperatorio, asintomático y puntuación AOFAS de 85. En el tobillo derecho, las puntuaciones fueron 75 y 90 , respectivamente. Tras 5 años de seguimiento, la paciente estaba asintomática de ambos tobillos, con una fuerza de flexión plantar de $5 / 5$, deambulación indolora y sin dificultad para subir las escaleras. No se observaron recidivas de las tumoraciones.

\section{Discusión}

Los xantomas pueden aparecer en las hiperlipidemias familiares severas. Otros tipos de xantomas, como el xantoma palmar o el xantoma eruptivo, pueden aparecer en pacientes con hipertrigliceridemia. El diagnóstico diferencial de los xantomas incluye los nódulos reumatoides, sarcomas o neurofibromatosis. ${ }^{9}$ Cuando los xantomas aparecen en el tendón de Aquiles y de forma bilateral se debe sospechar una xantomatosis cerebrotendinosa.

La xantomatosis cerebrotendinosa se caracteriza por la formación de xantomas en el tendón de Aquiles, cataratas bilaterales y deterioro neurológico progresivo. El primer síntoma suele ser la catarata bilateral. ${ }^{10-12}$ Los xantomas localizados en el tobillo son raros y suelen manifestarse en la mayoría de ocasiones como lesiones múltiples. El 50\% de los xantomas que aparecen en el tobillo se localizan en el tendón de Aquiles. ${ }^{13}$ En la literatura hay una falta de estudios sobre xantomatosis cerebrotendinosa diagnosticados tras la 
detección de xantomas del tendón de Aquiles. La mayoría de los casos de xantomatosis cerebrotendinosa que muestra la literatura, se diagnosticaron en pacientes menores de 30 años. La importancia del caso radica en su sospecha diagnóstica, especialmente tratándose de pacientes de mayor edad. A nuestro entender, solo se ha descrito un caso de xantomatosis cerebrotendinosa y xantomas en el tendón de Aquiles en un paciente de edad. ${ }^{14}$ Ese caso era un hombre de 63 años, diagnosticado tras la detección de un aneurisma de aorta y xantomas en ambos tendones de Aquiles y fascias plantares. Recibió tratamiento médico con estatinas, y quirúrgico del aneurisma, pero no se intervinieron los xantomas tendinosos dado que no producían manifestaciones clínicas.

Los xantomas tienden a recurrir si no se realiza la resección total del mismo, ${ }^{1}$ con una tasa de recurrencia tras la resección parcial ${ }^{13}$ entre un $12 \%$ y un $15 \%$. Debido a esto, Tomita y col., ${ }^{2}$ recomendaba la resección total del tendón de Aquiles entre la unión miotendinosa y el calcáneo para los pacientes jóvenes. En el presente caso, la resección fue parcial en ambos tendones de Aquiles, no habiendo recidivas después de cinco años.

Como conclusión, pensamos que la resección de la lesión puede ser parcial en los pacientes de edad, a pesar de que las tumoraciones infiltren extensamente al tendón de Aquiles, resultando en un excelente resultado funcional y sin aparición de recidivas.

Conflicto de Intereses

Los autores declaran no tener ningún conflicto de intereses.

\section{Referencias}

1 Dinçel YM, Arıkan Y, Özer D, Basılgan S. Reconstruction of the Achilles tendon using quadriceps tendon graft in bilateral xanthomas secondary to familial hypercholesterolemia: A case report. Eklem Hastalik Cerrahisi 2018;29(02):117-122
2 Tomita T, Ochi T, Fushimi H, Matsuzawa Y, Ono K. Reconstruction of the Achilles tendon for xanthoma: findings at operative reexploration. A case report. J Bone Joint Surg Am 1994;76(03):444-447

3 Griffith JF, Hu M, Yeung DKW, et al. Achilles tendon xanthomas: fat-water separation at baseline and after treatment. Radiology 2017;285(03):876-884

4 Abate M, Schiavone C, Salini V, Andia I. Occurrence of tendon pathologies in metabolic disorders. Rheumatology (Oxford) 2013; 52(04):599-608

5 Junyent M, Gilabert R, Zambón D, et al. The use of Achilles tendon sonography to distinguish familial hypercholesterolemia from other genetic dyslipidemias. Arterioscler Thromb Vasc Biol 2005; 25(10):2203-2208

6 Descamps OS, Leysen X, Van Leuven F, Heller FR. The use of Achilles tendon ultrasonography for the diagnosis of familial hypercholesterolemia. Atherosclerosis 2001;157(02):514-518

7 Jarauta E, Mateo-Gallego R, Bea A, Burillo E, Calmarza P, Civeira F. Carotid intima-media thickness in subjects with no cardiovascular risk factors. Rev Esp Cardiol 2010;63(01):97-102

8 Tsouli SG, Xydis V, Argyropoulou MI, Tselepis AD, Elisaf M, Kiortsis DN. Regression of Achilles tendon thickness after statin treatment in patients with familial hypercholesterolemia: an ultrasonographic study. Atherosclerosis 2009;205(01):151-155

9 Aljenedil S, Ruel I, Watters K, Genest J. Severe xanthomatosis in heterozygous familial hypercholesterolemia. J Clin Lipidol 2018; 12(04):872-877

10 Moghadasian MH. Cerebrotendinous xanthomatosis: clinical course, genotypes and metabolic backgrounds. Clin Invest Med 2004;27(01):42-50

11 Smithard A, Lamyman MJ, McCarthy CL, Gibbons CL, Cooke PJ, Athanasou N. Cerebrotendinous xanthomatosis presenting with bilateral Achilles tendon xanthomata. Skeletal Radiol 2007;36 (02):171-175

12 Mirzanli C, Esenyel CZ, Ozturk K, Baris A, Imren Y. Cerebrotendinous xanthomatosis presenting with bilateral achilles tendon xanthomata: a case report. J Am Podiatr Med Assoc 2013;103(02):152-155

13 Friedman MS. Xanthoma of the Achilles tendon. J Bone Joint Surg Am 1947;29(03):760-766

14 Tada H, Inaba S, Pozharitckaia D, Kawashiri MA. Prominent tendon xanthomas and abdominal aortic aneurysm associated with cerebrotendinous xanthomatosis identified using whole exome sequencing. Intern Med 2018;57(08):1119-1122 